Research Article

\title{
The Static and Dynamic Sensitivity of Magnetostrictive Bioinspired Whisker Sensor
}

\author{
Ran Zhao (iD, Qingjie Yuan, Jianwu Yan, and Qanguo Lu \\ Jiangxi Province Key Laboratory of Precision Drive \& Control, Nanchang Institute of Technology, Nanchang, China \\ Correspondence should be addressed to Ran Zhao; zhaoran@nit.edu.cn
}

Received 16 August 2017; Revised 13 November 2017; Accepted 27 November 2017; Published 29 January 2018

Academic Editor: Peng Zhang

Copyright (C) 2018 Ran Zhao et al. This is an open access article distributed under the Creative Commons Attribution License, which permits unrestricted use, distribution, and reproduction in any medium, provided the original work is properly cited.

\begin{abstract}
Magnetostrictive bioinspired whisker is a new kind of sensor that can realize tactile and flow sensing by utilizing magnetoelastic effect. The sensitivity is a key technical indicator of whisker sensor. The paper presented a new magnetostrictive whisker based on Galfenol cantilever beam, as well as its operation principle. Then, the static and dynamic sensitivity of the whisker sensor was investigated by using a self-made experimental system. The results illustrated that the proposed sensor has a high sensitivity. Its static sensitivity is $2.2 \mathrm{mV} / \mathrm{mN}$. However, its dynamic sensitivity depends on the vibration frequency. When working at the natural frequency of the cantilever beam, the dynamic sensitivity performs an obvious increase- $1.3 \mathrm{mV} / \mathrm{mN}$ at $3.5 \mathrm{~Hz}$ (the first-order natural frequency) and $2.1 \mathrm{mV} / \mathrm{mN}$ at $40 \mathrm{~Hz}$ (the second-order natural frequency), respectively.
\end{abstract}

\section{Introduction}

In nature, animals, such as seal and rat, use their whiskers to perceive the external environment. The position, shape, and surface texture of the object are sensed by the whisker and transmitted to its base. These stimulates are converted to bioelectrical signal and passed to the animal's brain $[1,2]$. Scholars utilize this principle to develop biomimetic whisker, applying them to tactile, shape, and flow sensing [3-5]. Recently, bioinspired whiskers have been served in robotics tactile, underwater vehicles, underwater structure detection, and other fields [6-9].

A typical bioinspired whisker consists of two main parts: sensing unit and measurement unit. The sensing unit is a cantilever beam similar to an animal's whisker structure. There are several materials used to fabricate the cantilever, such as elastic material (polymers), piezoelectric material (PZT-5 and PVDF), and magnetostrictive material (Galfenol and Alfenol) [10-14]. The working principles of these sensors are different from each other. Polymer whiskers are based on the measure of beam's bending angle. However, piezoelectric whiskers rely on the inverse piezoelectric effect. Magnetostrictive whiskers use magnetoelastic effect (or Villari effect) to achieve force perception. Among the three kinds of whiskers, magnetostrictive whisker has the advantages of simple structure, robotic, and the coupling measurement for dynamic and static conditions $[15,16]$. Therefore, it has better application prospect and research value.

For magnetostrictive whisker, its stress-induced bending results in magnetic flux density changes of magnetostrictive material, which is detected by Hall Effect sensors. With this principle, the static and dynamic stress can be perceived. Sensitivity is the key technical parameter for a whisker sensor, which determines the sensor's measuring range and accuracy. Therefore, we established an experimental system to test the static and dynamic responses of the magnetostrictive whisker sensor. With the experimental data, the paper also studied the influence of stress and vibration frequency to sensitivity.

\section{Principle}

Figure 1 shows the construction of a magnetostrictive whisker. There are four parts consisting of the proposed whisker (Figure 1(a)): magnetostrictive cantilever beam, bias magnet, Hall Effect sensor, and housing. The bias magnet is fixed to the end of the beam to apply a bias magnetic field. The Hall Effect sensor is put under the cantilever to detect 


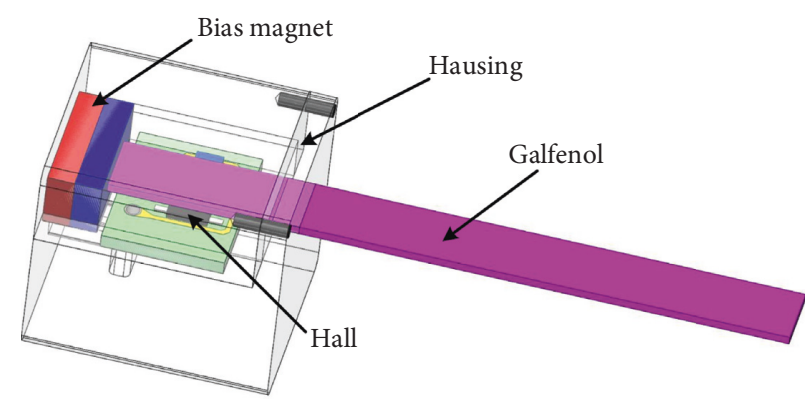

(a)

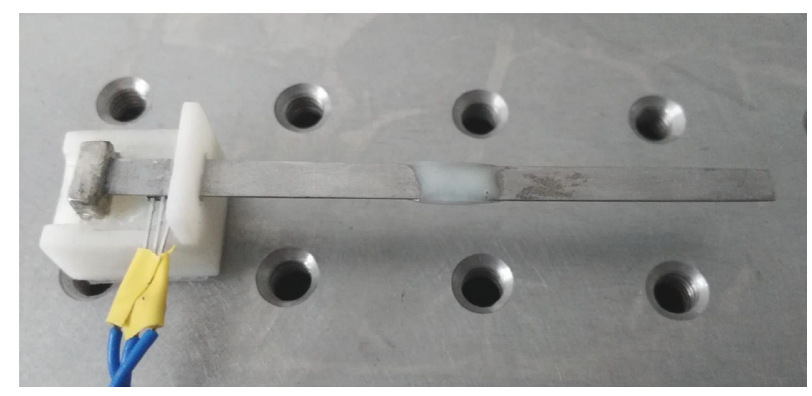

(b)

FIGURE 1: (a) 3D model and (b) photograph of Galfenol whisker sensor.

TABLE 1: Physical and geometrical parameters of Galfenol whisker sensor.

\begin{tabular}{lccc}
\hline Material & Parameter name & Symbol & Value \\
\hline \multirow{2}{*}{ Bias magnet } & Magnetic field & $H_{c}$ & $300 \mathrm{mT}$ \\
& Dimension & Length $\times$ width $\times$ high & $8.5 \mathrm{~mm} \times 3.5 \mathrm{~mm} \times 2 \mathrm{~mm}$ \\
Galfenol & Saturation magnetostriction & $\lambda_{s}$ & $170 \mathrm{ppm}$ \\
& Young's modulus & $E$ & $85 \mathrm{GPa}$ \\
Hall & Poisson's ratio & $v$ & 0.35 \\
& Dimension & Length $\times$ width $\times$ high & $80 \mathrm{~mm} \times 4 \mathrm{~mm} \times 0.3 \mathrm{~mm}$ \\
\hline
\end{tabular}

the change of magnetic field. Here, based on the magnetoelastic effect, it will generate flux change in the bending beam. The leakage flux will change simultaneously, and this change can be measured by the Hall or GMR sensor. It means the utilization of magnetoelastic effect is indirect. That is the difference of magnetostrictive whisker sensor to conventional magnetostrictive force sensor and energy harvester.

Figure 1(b) shows the photograph of the magnetostrictive whisker sensor. In this work, the rolled Galfenol sheet is used to fabricate the magnetostrictive cantilever beam. It has good flexibility, high Young's modulus, and magnetostriction of $140-180$ ppm $[17,18]$. The physical and geometrical parameters of the magnetostrictive whisker sensor and bias magnet are given in Table 1.

\section{Experiment}

We established an experimental system to test the performance of the magnetostrictive whisker. Figure 1(a) shows the static experiment. The Galfenol cantilever beam bends when weights are hanged on its tips. The voltage-force curve is obtained with the static stress. The displacement of the whisker's tip is also measured to obtain the relation between stress and deflection. Figure 1(b) shows the dynamic test equipment. In this system, a sinusoidal signal is generated by dSPACE and amplified by the amplifier to drive the vibration exciter. This sinusoidal excitation is used to simulate the vibration from nature. The vibration frequency is set in the range of $0.5-60 \mathrm{~Hz}$. Besides, the dynamic deflection is measured by the laser displacement sensor. Data from the Hall sensor and laser sensor are obtained by dSPACE platform.
Because the dynamic stress is very different to measure, we utilize a conversion coefficient $\gamma$ to convert the dynamic displacement into dynamic stress. This coefficient is defined as the ratio of stress to displacement, given by

$$
\gamma=f\left(\frac{\sigma}{u}\right)
$$

where $u$ is the displacement of whisker tip, $\sigma$ represents stress, and $\gamma$ is expressed as a function about $u$ and $\sigma$. Its specific form can be obtained through the experiment.

For a measurement system consisting of multiple units, assuming the sensitivity of its parts as $s_{1}, s_{2}, s_{3}, \ldots, s_{n}$, respectively, the total sensitivity of this system can be expressed as $s=s_{1} s_{2} s_{3}, \ldots, s_{n}$. There is a slight difference between the static sensitivity and dynamic sensitivity. The static sensitivity $\left(s_{s}\right)$ of the magnetostrictive whisker sensor is defined as voltage versus stress, which is

$$
s_{s}=s_{G} s_{H}=\frac{\Delta B}{\Delta \sigma} \cdot \frac{\Delta V}{\Delta B}=\frac{\Delta V}{\Delta \sigma}
$$

where $s_{G}$ represents the sensitivity of Galfenol beam and $s_{H}$ is the sensitivity of the Hall sensor. $\Delta B$ is the change of magnetic flux density, $\Delta \sigma$ is the change of stress, and $\Delta V$ is the change of voltage. Therefore, the dynamic sensitivity $s_{d}$ can be expressed as

$$
s_{d}=s_{G} s_{H}=\frac{d B}{d \sigma} \cdot \frac{d V}{d B}=\frac{d V}{d \sigma},
$$

where $d B / d \sigma$ represents the derivative of magnetic flux density to stress, and $d V / d B$ is the derivative of voltage to magnetic flux density. 


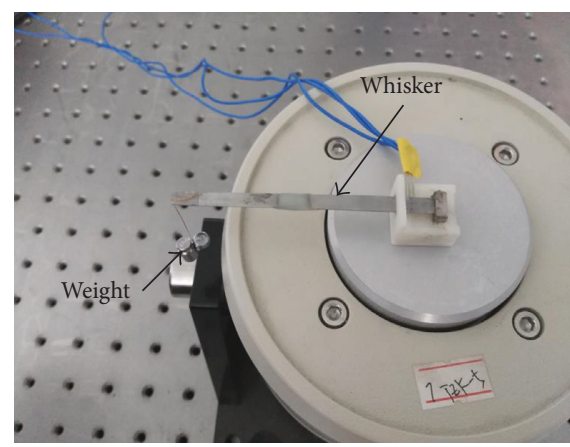

(a)

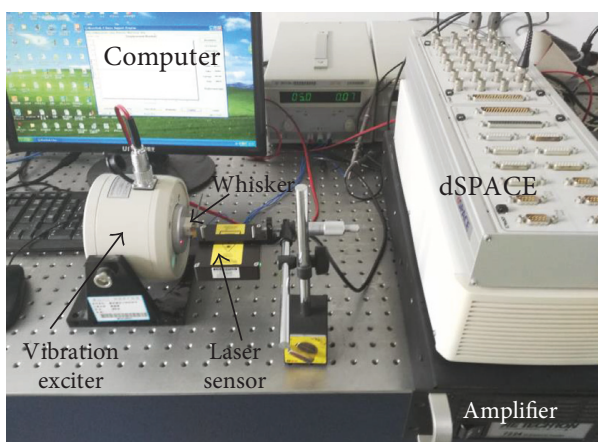

(b)

Figure 2: Experimental system for (a) static bending test and (b) vibration test.

\section{Results and Discussion}

4.1. Static Sensitivity. With the method of hanging weights (Figure 2(a)), the static response of the magnetostrictive whisker sensor is obtained and displayed in Figure 3. The weights are changing from 3 to $33 \mathrm{~g}$, which means the static stress acting on the cantilever beam changes from 0.029 to $0.323 \mathrm{~N}$. When the load is $0.323 \mathrm{~N}$, the whisker reached its maximum allowable deflection. The discrete data are fitted by a fifth-order polynomial and is shown in Figure 3. This polynomial is expressed as

$$
s_{S}(\sigma)=5 \sigma^{5}+50 \sigma^{4}-63.25 \sigma^{3}-20.51 \sigma^{2}+0.1668 \sigma,
$$

where $s_{s}(\sigma)$ is a function depends that on the varying stress.

The slope of voltage-force curve is defined as the sensitivity of the magnetostrictive whisker sensor. From the diagram, we found that the curve is almost a straight line, meaning that the linearity of static sensitivity is good. Therefore, the average static sensitivity here can be used instead of the actual static sensitivity. It can be defined as

$$
\bar{s}_{s}=\frac{\int_{0}^{\sigma_{m}} s_{s}(x) d x}{\sigma_{m}},
$$

where $\sigma_{m}$ is the maximum stress loading on the cantilever beam in the experiment. According to (4) and (5), the calculation value of static sensitivity $s_{s}$ is $2.20 \mathrm{mV} / \mathrm{mN}$.

Figure 4 shows the stress dependence of displacement, which is the deflection of the whisker tip. From the figure, we can see that the curve has good linearity. So, the function of

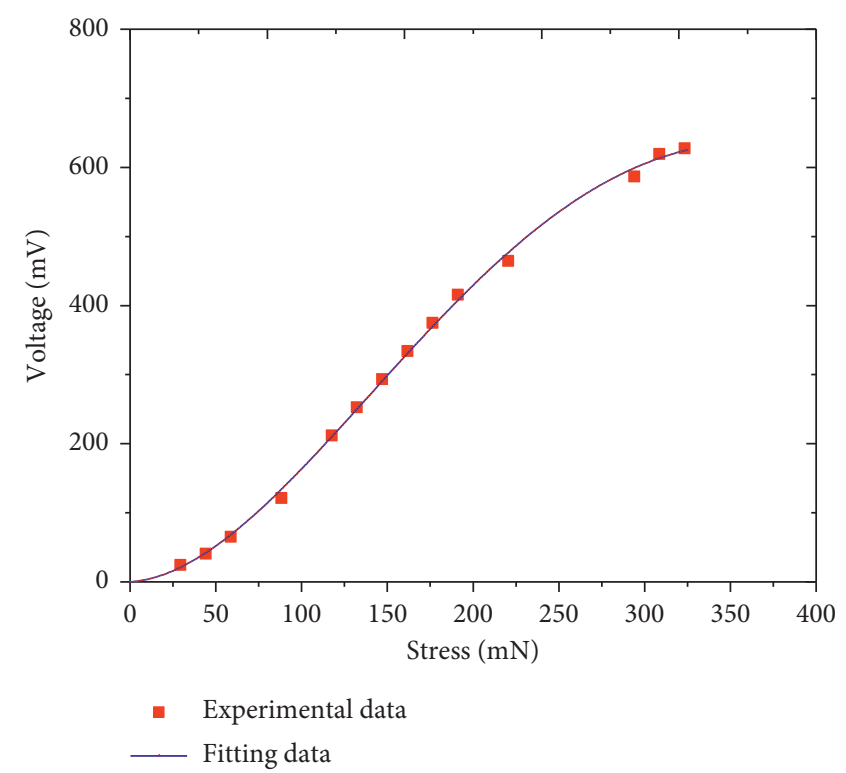

FIgURE 3: Static response of magnetostrictive whisker sensor with stress from 0.029 to $0.323 \mathrm{~N}$.

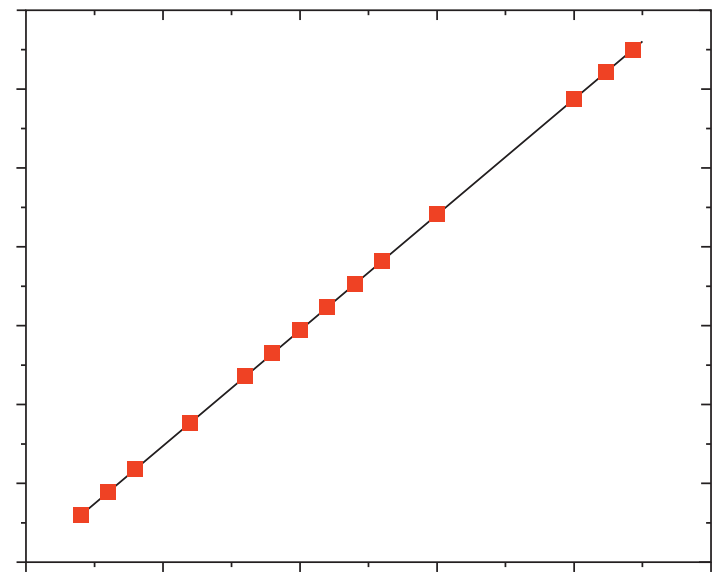

FIgUre 4: Curve of stress dependence on deflection.

conversion coefficient can be simplified to $\gamma=\sigma / u$. Therefore, $\gamma=14.7 \mathrm{mN} / \mathrm{mm}$.

4.2. Dynamic Sensitivity. Experimental results in Section 4.1 show that the change of stress (or the deflection of cantilever tip) has little influence on the sensitivity. Therefore, in dynamic testing, it is reasonable to assume that the sensitivity does not vary with amplitude of vibration (or dynamic stress). Based on it, when considering the dynamic response, we simplify the problem to research the relationship between the dynamic sensitivity and the vibration frequency.

Figure 5 shows the dynamic response of magnetostrictive whisker sensor when exciting frequency is changing from 0.5 to $60 \mathrm{~Hz}$. In Figure 5(a), the whisker sensor works at $0.5 \mathrm{~Hz}$, a $180^{\circ}$ phase difference is observed between the voltage and vibration signal. This opposite trend is because 


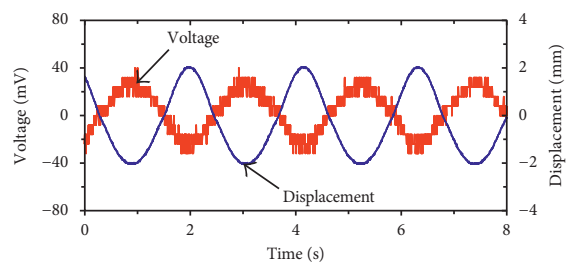

(a)

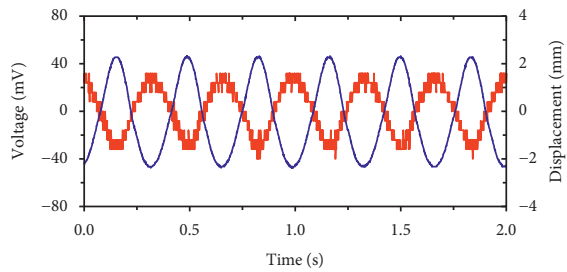

(d)

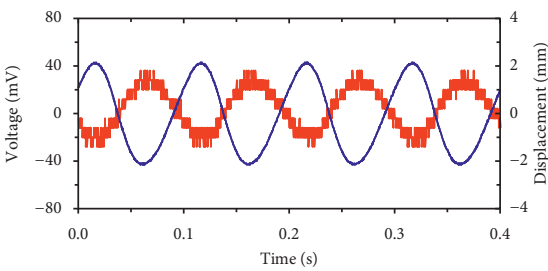

(g)

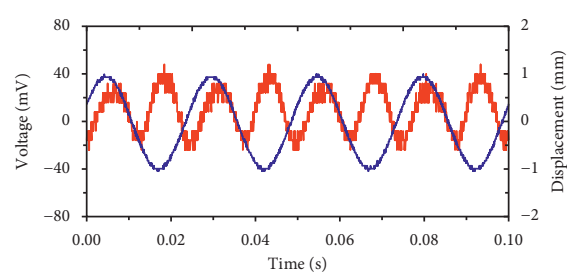

(j)

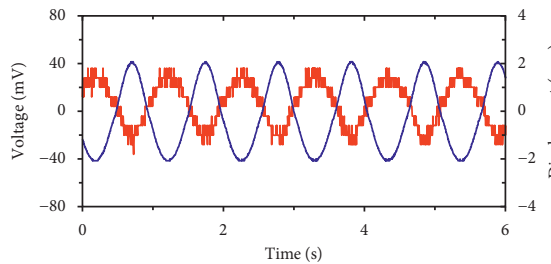

(b)

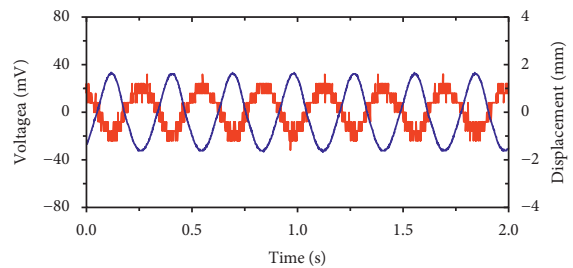

(e)

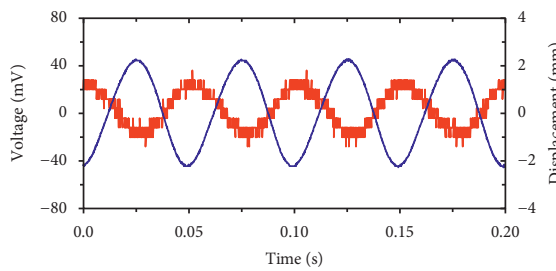

(h)

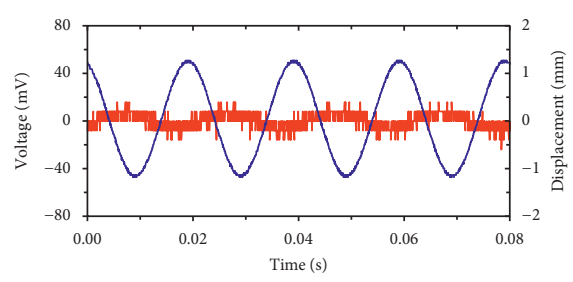

(k)

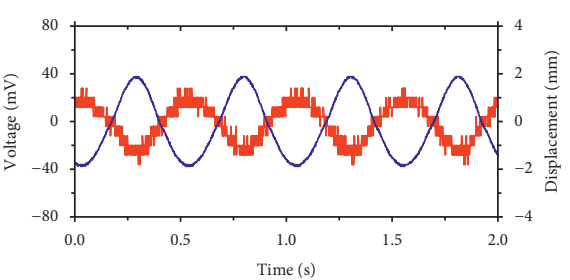

(c)

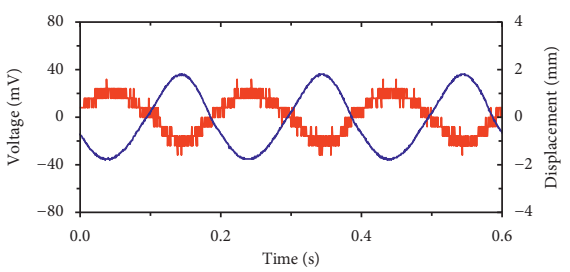

(f)

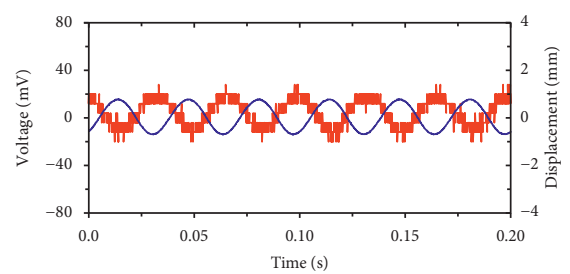

(i)

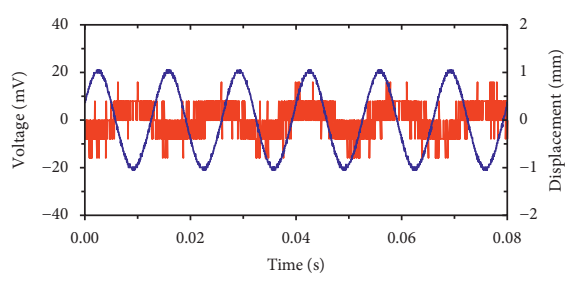

(l)

Figure 5: Dynamic response of magnetostrictive whisker sensor at exciting frequency of (a) 0.5, (b) 1, (c) 2, (d) 3, (e) 3.5, (f) 5, (g) 10, (h) 20, (i) 30, (j) 40, (k) 50, and (l) $60 \mathrm{~Hz}$.

that the change of leakage flux density is opposite to the density of the magnetic flux inside the material (Galfenol beam). In addition, another phenomenon is found, phase difference decreases with the increasing frequency. This is due to the elastic hysteresis of cantilever beam and the magnetic hysteresis of Galfenol material.

The responses are tested when the whisker sensor operates at its first- and second-order natural frequency $(3.5$ and $40 \mathrm{~Hz}$, resp.) (Figures 5(d) and 5(j)). When working at a frequency of $3.5 \mathrm{~Hz}$, the ratio of voltage to displacement has an obvious increase comparing to the working frequencies nearby. Furthermore, at $40 \mathrm{~Hz}$, the voltage frequency is two times as much as the exciting frequency. It confirms that $40 \mathrm{~Hz}$ is very close to second-order natural frequency of the cantilever beam. By using the conversion coefficient $\gamma$, we can convert the displacement (given in Figure 5) data into stress data, and then utilize (3) to calculate the dynamic sensitivity.
With the increasing vibration frequency, the response capability of the whisker sensor gradually declines. Figure $5(\mathrm{k})$ shows when the vibration frequency is $50 \mathrm{~Hz}$ and amplitude is $4.8 \mathrm{~mm}$, the amplitude of output voltage is $0.015 \mathrm{~V}$. Under the condition of $60 \mathrm{~Hz}$ frequency and $0.32 \mathrm{~mm}$ vibration amplitude, the amplitude of output voltage is $0.017 \mathrm{~V}$. These results illustrate that when operating frequency deviates from the cantilever beam's natural frequency, its response amplitude (output voltage) will be reduced.

Figure 6 shows the sensitivity-frequency curve of magnetostrictive whisker sensor. The dynamic sensitivity shows an obvious increase when working near the natural frequencies. When working at $0.5 \mathrm{~Hz}$, the sensitivity is $2.13 \mathrm{mV} / \mathrm{mN}$. Between 0 and $10 \mathrm{~Hz}$, the sensitivity curve reaches its first peak $-2.60 \mathrm{mV} / \mathrm{mN}$ at $3.5 \mathrm{~Hz}$. The value of sensitivity at first-order nature frequency is 1.1 times of that at $0.5 \mathrm{~Hz}$. Within the range of $20-60 \mathrm{~Hz}$, the 


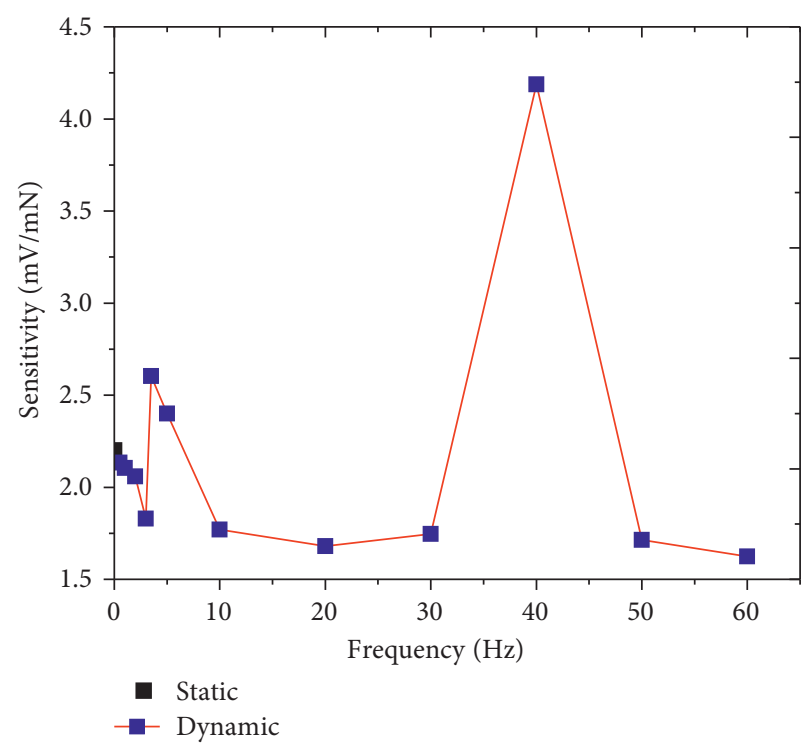

FIGURE 6: Sensitivity dependence of varying vibration frequency.

sensitivity curve reaches its second peak $-4.19 \mathrm{mV} / \mathrm{mN}$ at $40 \mathrm{~Hz}$. The value of sensitivity at second-order nature frequency reaches two times of that at $0.5 \mathrm{~Hz}$. We can conclude that the operating frequency has a great influence on the dynamic sensitivity of sensor.

\section{Conclusions}

In this work, we developed a Galfenol-based whisker sensor and established an experimental system to investigate its sensitivity. The static and dynamic performances of the magnetostrictive whisker sensor were obtained in the test. With these experimental results, we analyzed the influence of stress and vibration frequency on its sensitivity. Finally, the following conclusions were concluded:

(i) Under static condition, the sensitivity of the sensor is less affected by stress changes, which means the sensor has good linearity (with its static sensitivity $2.20 \mathrm{mV} / \mathrm{mN}$ ).

(ii) Under dynamic condition, the sensitivity of the sensor is greatly affected by the vibration frequency. The dynamic sensitivity curve shows two peaks in the range from 0.5 to $60 \mathrm{~Hz}$, when working at 3.5 and $40 \mathrm{~Hz}$, respectively.

(iii) The sensitivity can be greatly improved by making the sensor to work near its natural frequencies.

\section{Conflicts of Interest}

The authors declare that there are no conflicts of interest.

\section{Acknowledgments}

This work was supported by the Science \& Technology Project of Jiangxi Province Education Department of China (Grant no. GJJ161105), University Science \& Technology Projects of Jiangxi Province (Grant no. KJLD14096), and the
Open Fund of Jiangxi Province Key Laboratory of Precision Drive \& Control (Grant no. KFKT-201617).

\section{References}

[1] Q. Kan, R. Rajan, J. Fu, G. Kang, and W. Yan, "Elastic modulus of rat whiskers -A key biomaterial in the rat whisker sensory system," Materials Research Bulletin, vol. 48, no. 12, pp. 5026-5032, 2013.

[2] F. A. Lucianna, A. L. Albarracín, S. M. Vrech, F. D. Farfán, and C. J. Felice, "The mathematical whisker: a review of numerical models of the rat's vibrissa biomechanics," Journal of Biomechanics, vol. 49, no. 10, pp. 2007-2014, 2016.

[3] H. Beem, M. Hildner, and M. Triantafyllou, "Calibration and validation of a harbor seal whisker-inspired flow sensor," Smart Materials And Structures, vol. 22, no. 1, p. 014012, 2013.

[4] P. V. Alvarado and S. Bhat, "Whisker-like sensors with tunable follicle sinus complex for underwater applications," Bioinspiration, Biomimetics and Bioreplication, vol. 9055, p. 90550C, 2014.

[5] M. Kaneko and T. Tsuji, "A whisker tracing sensor with $5 \mu \mathrm{m}$ sensitivity," in Proceedings of IEEE International Conference on Robotics \& Automation, vol. 4, pp. 3907-3912, Brisbane, Australia, May 2000.

[6] M. Kaneko and T. Tsuji, "A whisker tracing sensor for manufacturing application," in Proceedings of IFAC Conference on Mechatronic Systems, pp. 383-388, Sydney, Australia, September 2000.

[7] F. Ju and S. F. Ling, "Bioinspired active whisker sensor for geometry detection of high aspect ratio microholes with simultaneous actuation and sensing capability," Smart Materials and Structures, vol. 24, no. 3, p. 035010, 2015.

[8] F. P. Torgal, J. A. Labrincha, M. V. Diamanti, C.-P. Yu, and H. K. Lee, Biotechnologies and Biomimetics for Civil Engineering, pp. 255-274, Springer International Publishing, Cham, Switzerland, 2013.

[9] S. R. Day, A. B. Flatau, S. M. Na, and R. A. Swartz, "The design and construction of a scour monitoring system," in SPIE Proceedings of Sensors and Smart Structures Technologies for Civil, Mechanical and Aerospace Systems, vol. 9061, p. 90610F, San Diego, CA, USA, May 2014.

[10] J. Wang, M. Kimura, and M. Taya, "Bio-inspired design of tactile sensors based on ionic polymer metal composites," in Proceedings of 17th International Conference on Composite Materials, vol. B4, pp. 1-8, Edinburgh, UK, July 2009.

[11] P. V. Alvarado, V. Subramaniam, and M. Triantafyllou, "Performance analysis and characterization of bio-Inspired whisker sensors for underwater applications," in Proceedings of IEEE International Conference on Intelligent Robots and Systems, vol. 8215, no. 2, pp. 5956-5961, Vilamoura-Algarve, Portugal, October 2013.

[12] F. Ju and S. F. Ling, "Bio-inspired active whisker sensor for robotic vibrissal tactile sensing," Smart Materials and Structures, vol. 23, no. 12, p. 125003, 2014.

[13] M. I. Tiwana, S. J. Redmond, N. H. Lovel, and J. Iqbal, "Bioinspired PVDF-based, mouse whisker mimicking, tactile sensor," Applied Sciences, vol. 6, no. 10, pp. 297-312, 2016.

[14] G. Raghunath, A. B. Flatau, S. M. Na, and B. Barkley, "Development of a bio-inspired tactile magnetostrictive whisker sensor using alfenol," in Proceedings of the ASME Conference on Smart Materials, Adaptive Structures and Intelligent Systems, pp. V002T04A012-V002T04A018, Snowbird, UT, USA, September 2014.

[15] P. R. Downey and A. B. Flatau, "Bending behavior of irongallium (Galfenol) alloys for sensor applications," in SPIE 
Proceedings of Sensors and Smart Structures Technologies for Civil, Mechanical, and Aerospace Systems, vol. 5764, pp. 120-129, San Diego, CA, USA, May 2005.

[16] S. Datta and A. B. Flatau, "Application of iron-gallium alloy as magnetostrictive sensors," in Proceedings of SPIE Conference on Smart Structures and Integrated Systems, vol. 5764, no. 4, pp. 720-729, San Diego, CA, USA, March 2005.

[17] R. Meloy and E. Summers, "Magnetic property-texture relationships in Galfenol rolled sheet stacks," Journal of Applied Physics, vol. 109, no. 7, pp. 8621-8624, 2011.

[18] H. Chun, S. M Na, J. H. Yoo, M. Wuttig, and A. B. Flatau, "Tension and strain annealing for abnormal grain growth in magnetostrictive Galfenol rolled is better sheet," Journal of Applied Physics, vol. 109, no. 7, pp. 4132-4135, 2011. 


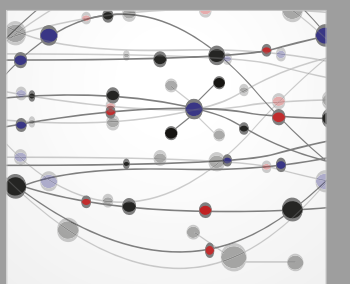

The Scientific World Journal
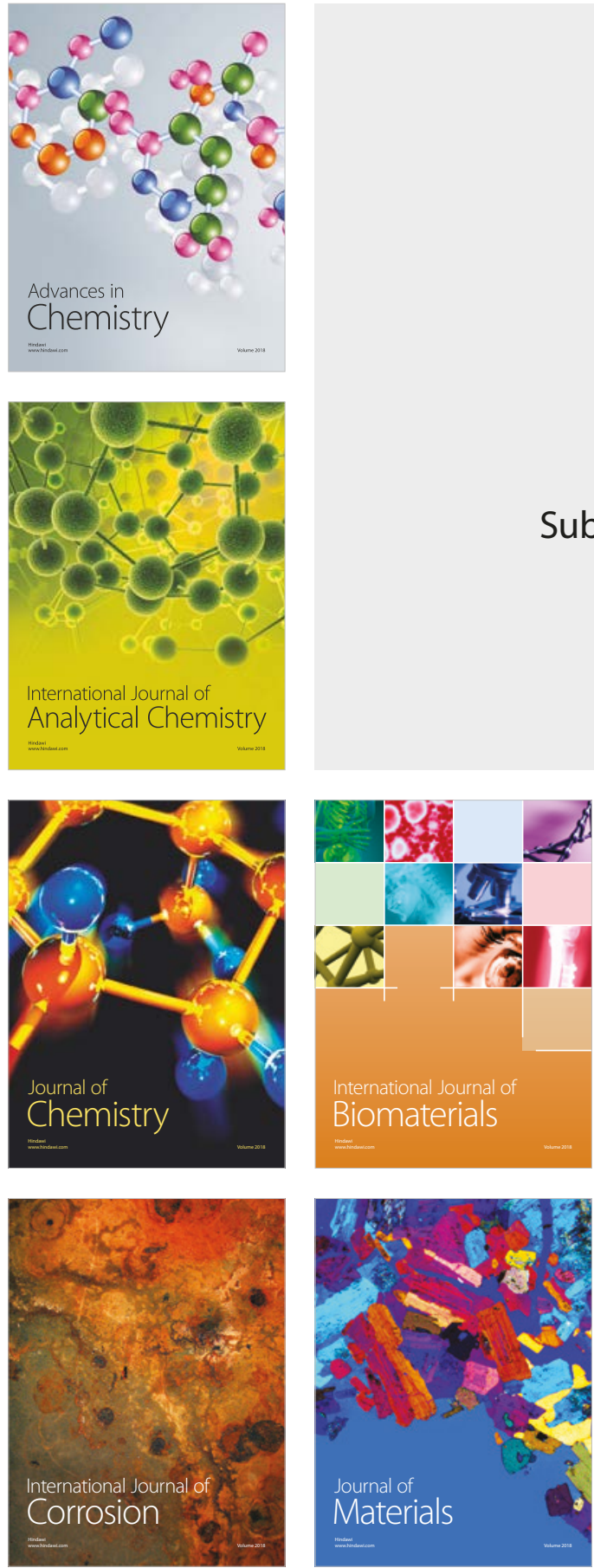

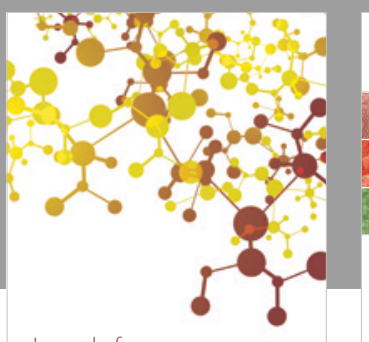

Journal of

Applied Chemistry
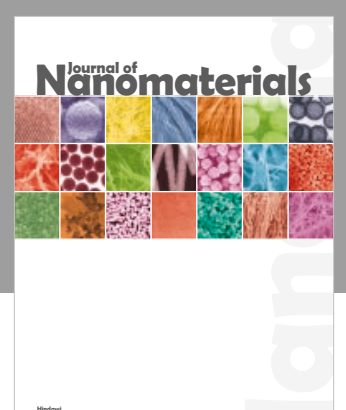

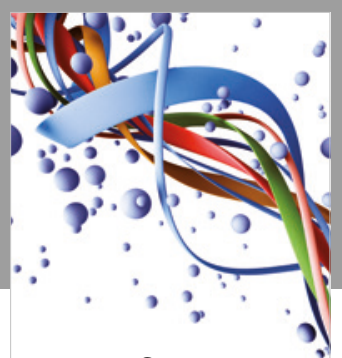

Scientifica

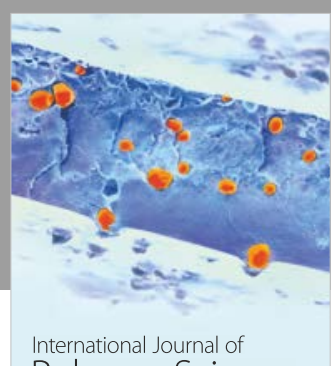

Polymer Science

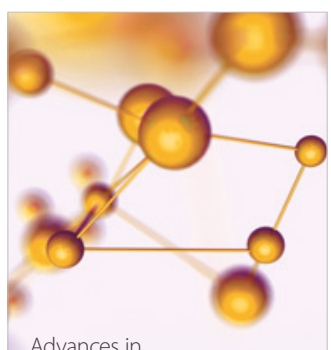

Physical Chemistry
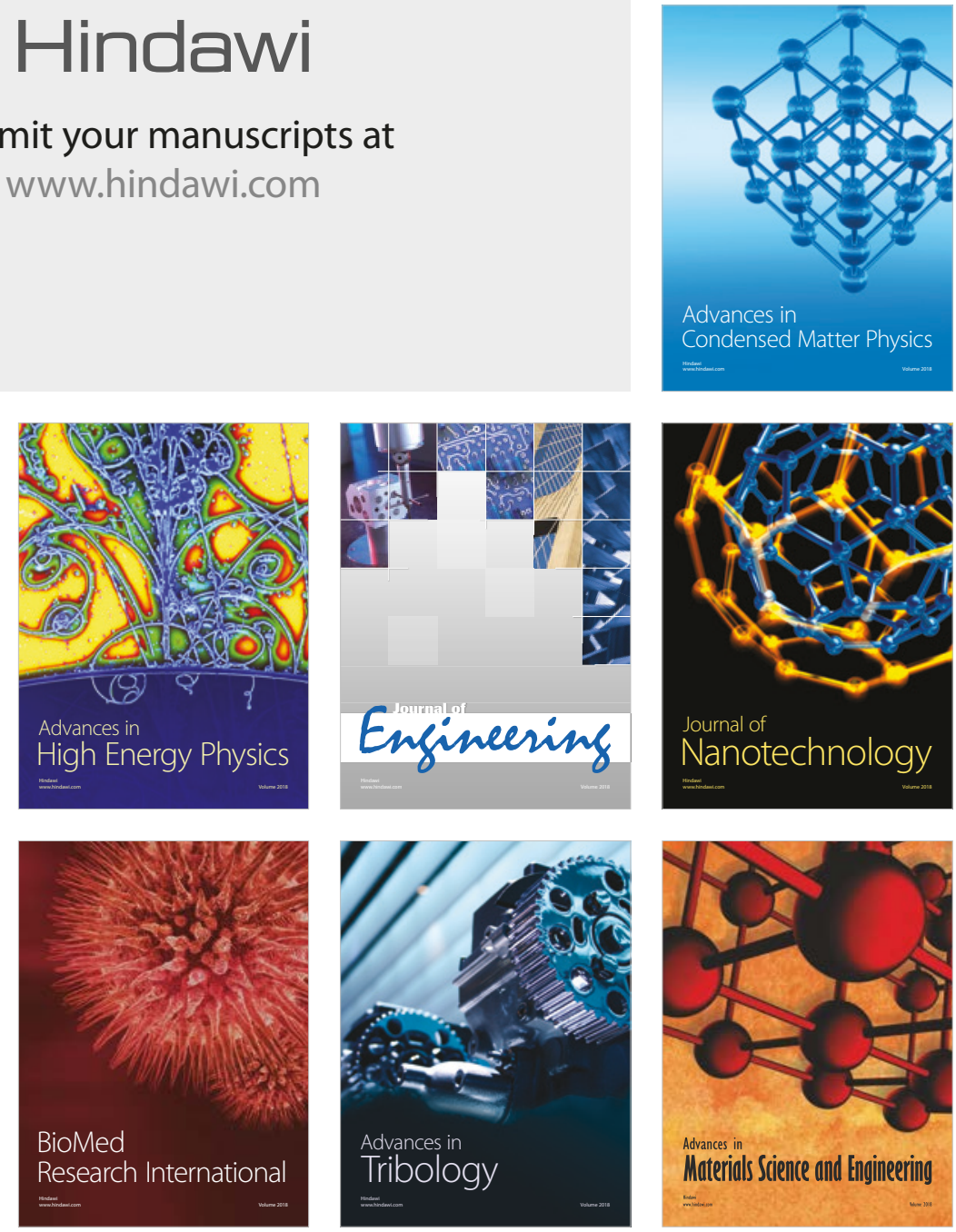